Supporting Information for

\title{
Heterointerface-Enhanced Ultrafast Optical Switching via Manipulating Metamaterial-Induced Transparency in a Hybrid Terahertz Graphene Metamaterial
}

Yuwang Deng, ${ }^{\dagger}$ Qingli Zhou, ${ }^{*}{ }^{\dagger}$ Pujing Zhang, ${ }^{\dagger}$ Nan Jiang, ${ }^{\dagger}$ Tingyin Ning, ${ }^{*}$ „ Wanlin Liang, ${ }^{\dagger}$ and Cunlin Zhang

${ }^{\dagger}$ Key Laboratory of Terahertz Optoelectronics, Ministry of Education, and Beijing Advanced Innovation Center for Imaging Theory and Technology, Department of Physics, Capital Normal University, Beijing 100048, China

‡Shandong Provincial Engineering and Technical Center of Light Manipulations \& Shandong Provincial Key Laboratory of Optics and Photonic Device, School of Physics and Electronics, Shandong Normal University, Jinan 250358, China

*E-mail: qlzhou@cnu.edu.cn (Q. Z.).

*E-mail: ningtingyin@sdnu.edu.cn (T.N.).

\section{Fabrication process of ULDB/GaAs:}

Periodic arrays of ULDB were fabricated on semi-insulating GaAs substrates using standard photolithographic methods. During this process, a layer of photoresist was spin-coated on the substrate surface, and ultraviolet exposure technology was used to form structure pattern. A $200 \mathrm{~nm}$ thick layer of gold (Au) with a $5 \mathrm{~nm}$ chrome (Cr) adhesive layer was evaporated on top of the film layer by the magnetron sputtering. The $\mathrm{Cr} / \mathrm{Au}$ layer was then patterned by the 
lift-off. Example optical images are shown in Figure S1 for the fabricated metasurface structures.

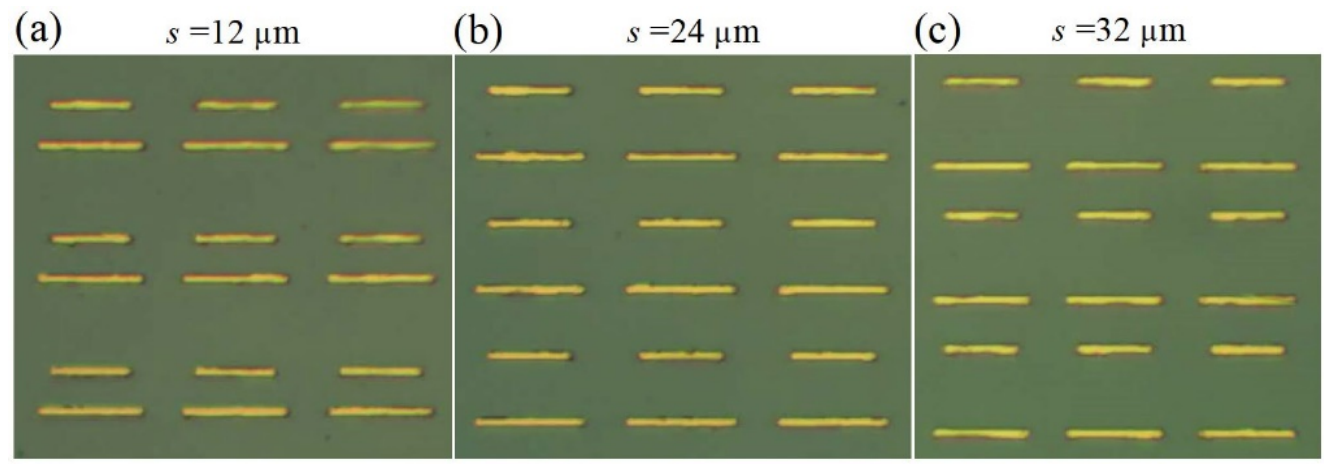

Figure S1. Optical photos of three ULDB/GaAs samples with different bar spacing $s$.

\section{Growth and transfer of graphene:}

The large-area single layer graphene film was prepared by CVD method on $25 \mu \mathrm{m}$ copper foil. Before the growth, the copper foil was heated in vacuum with 20 sccm hydrogen from room temperature to $1,000^{\circ} \mathrm{C}$ for removal of surface oxides. As the temperature reached $1,000^{\circ} \mathrm{C}$, a mixed flow of 2 sccm $\mathrm{H}_{2}$ and 5 sccm $\mathrm{CH}_{4}$ was introduced for graphene growth and this monolayer graphene growth time was 10 mins. Then the graphene was transferred onto ULDB/GaAs metamaterials using polymethyl methacrylate (PMMA)-assisted method. Firstly, the graphene film on $\mathrm{Cu}$ foil was spin-coated with PMMA. Secondly, $2 \mathrm{~mol} / \mathrm{L} \mathrm{FeCl}_{3}$ solution was used to etch away the $\mathrm{Cu}$ substrate, resulting in a free-standing PMMA/graphene film floating on the surface of the solution. Thirdly, the PMMA/graphene was cleaned out by deionized water for 4 times. Finally, it was transferred onto ULDB/GaAs sample and the acetone solution was used to dissolve the PMMA. The same method was used to cover graphene films on $\mathrm{SiO}_{2}$ and $\mathrm{GaAs}$ substrates, respectively. 


\section{Electrical field distribution:}

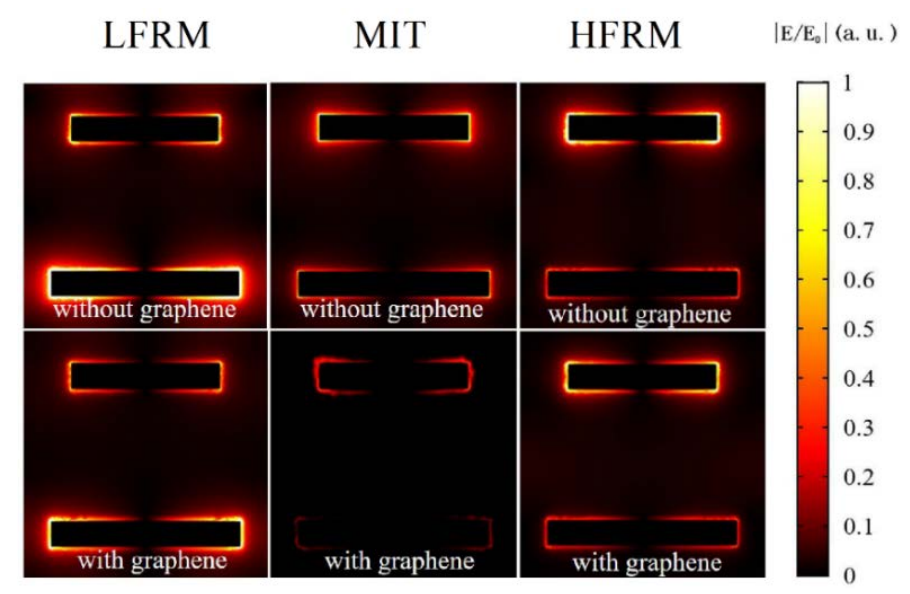

Figure S2. Simulated electric field distributions at LFRM, MIT, and HFRM of ULDB/GaAs sample without and with monolayer graphene overlayer for $s$ of $32 \mu \mathrm{m}$.

\section{Measured relative change in THz peak transmission}

As shown in Figure S3a and b, the pump-induced change in THz transmission increases with pump power. For each curve, if the THz signal is ahead of the pump pulse, i.e., the delay time is negative, $\Delta T / T_{0}$ will be zero due to the unchanged $\mathrm{THz}$ peak value. When the $\mathrm{THz}$ pulse encounters the pump pulse, the $\mathrm{THz}$ transmission decreases dramatically due to the photoexcited carriers in the hybrid structure, resulting in the negative value of $\Delta T / T_{0}$. However, for the known p-doped CVD graphene sample, we observe a positive $\Delta T / T_{0}$ that increases monotonically with pump fluence presented in Figure S3c. It has been well explained that the sign of $\Delta T / T_{0}$ depends on the doping concentration. ${ }^{1-4}$ This behavior is attributed to suppression of the graphene photoconductivity due to an increase in the carrier scattering rate induced by the increase in the photogenerated carrier concentration, resulting in metal-like properties of highly photo-doped graphene. ${ }^{5}$ For the same graphene/ $\mathrm{SiO}_{2}$ sample exposed to the air, this pump-induced transmission is much higher than that in the nitrogen environment, 
which is ascribed to that the increasing adsorption of oxygen and water vapor has an impact on increasing Fermi energy to tune the positive relative change in THz peak transmission, as given in Figure S3d. Here, it is worth noting that if the graphene is transferred onto GaAs surface to form a heterostructure, the magnitude of negative $\Delta T / T_{0}$ in graphene/GaAs is even larger than that of the bare GaAs substrate due to the effective photoexcited carrier separation through the heterointerface.
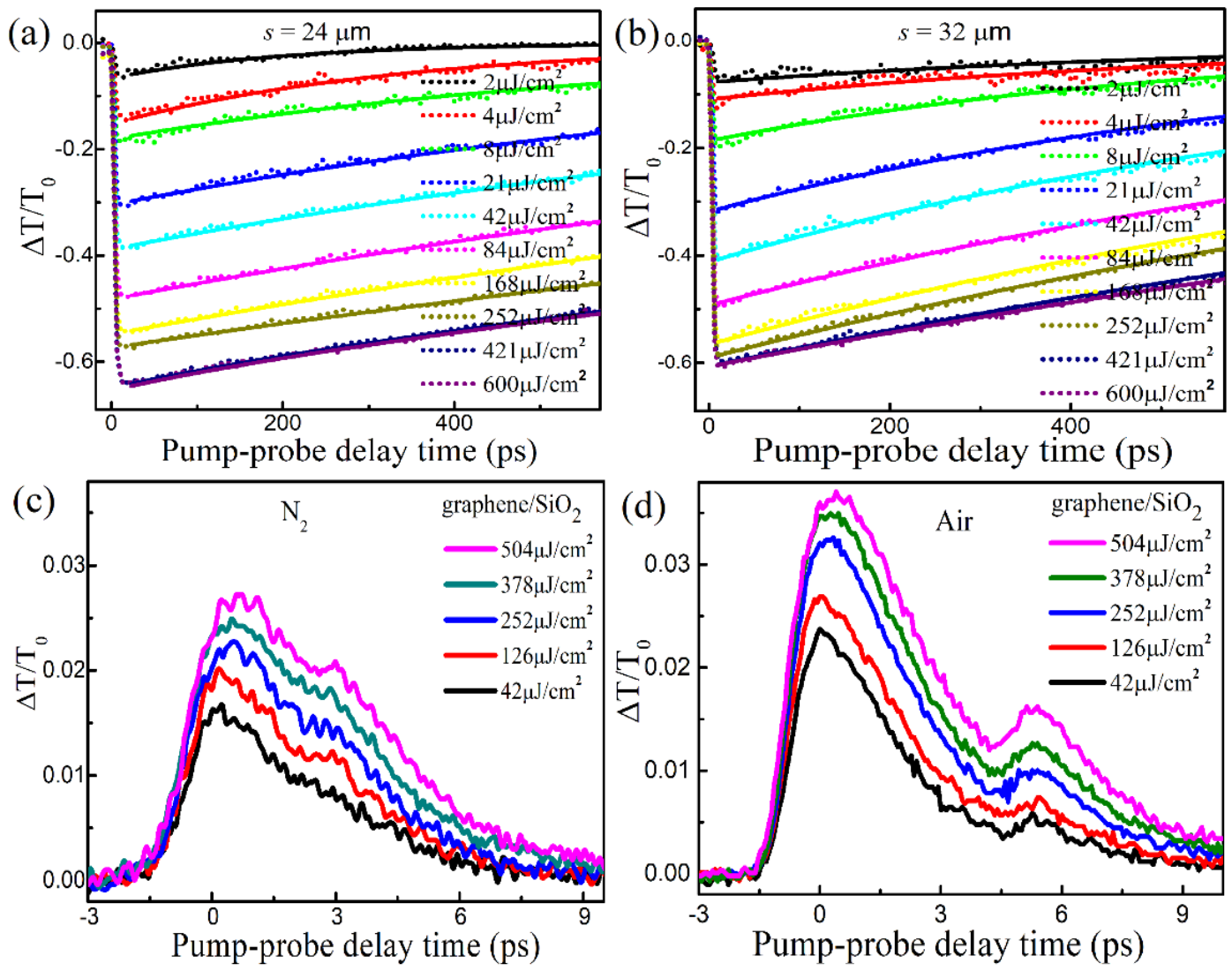

Figure S3. (a) and (b) are the measured photoexcited dynamics (dotted line) of the hybrid metamaterials performed by the OPTP measurement with different pump fluences for $s$ of 24 and $32 \mu \mathrm{m}$, respectively. Solid lines are the biexponential fitting. (c) and (d) are the measured photoexcited dynamic of graphene on $\mathrm{SiO}_{2}$ substrate in $\mathrm{N}_{2}$ and air, respectively 


\section{Ultrafast THz spectral evolution}

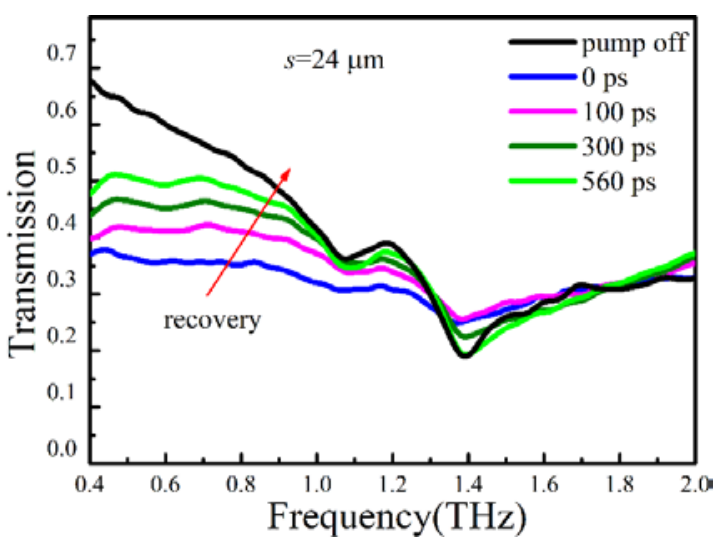

Figure S4. Ultrafast THz spectral evolution of hybrid metamaterial for $s$ of $24 \mu \mathrm{m}$ at different optical pump delay time under pump fluence of $2 \mu \mathrm{J} / \mathrm{cm}^{2}$.

\section{Transmission curves}
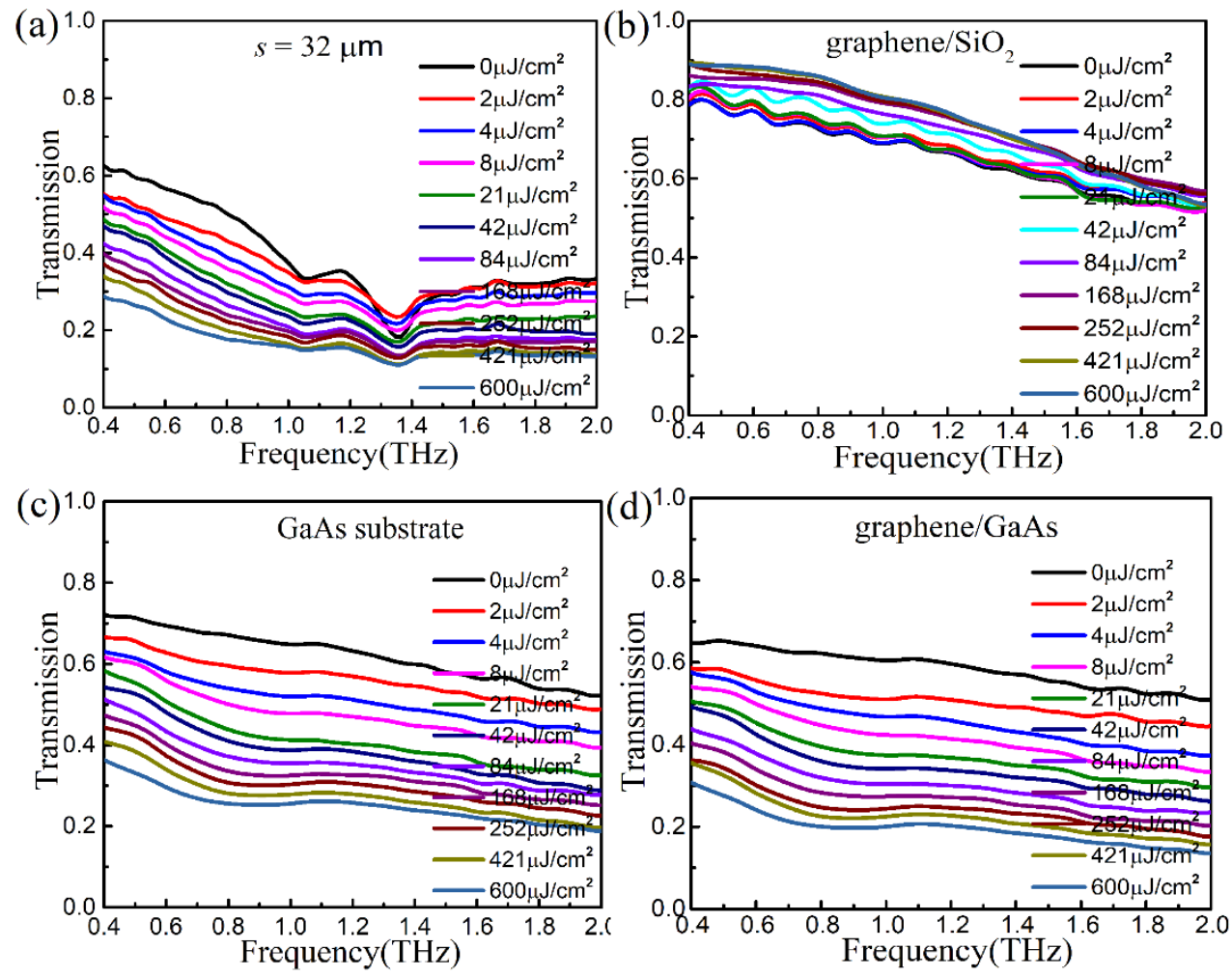

Figure S5. The amplitude transmission curves as function of pump fluence for hybrid sample

with $s$ of $32 \mu \mathrm{m}$ (a), graphene/SiO 2 (b), GaAs substrate (c) and graphene/GaAs (d), respectively. 


\section{Simulated transmission spectra}
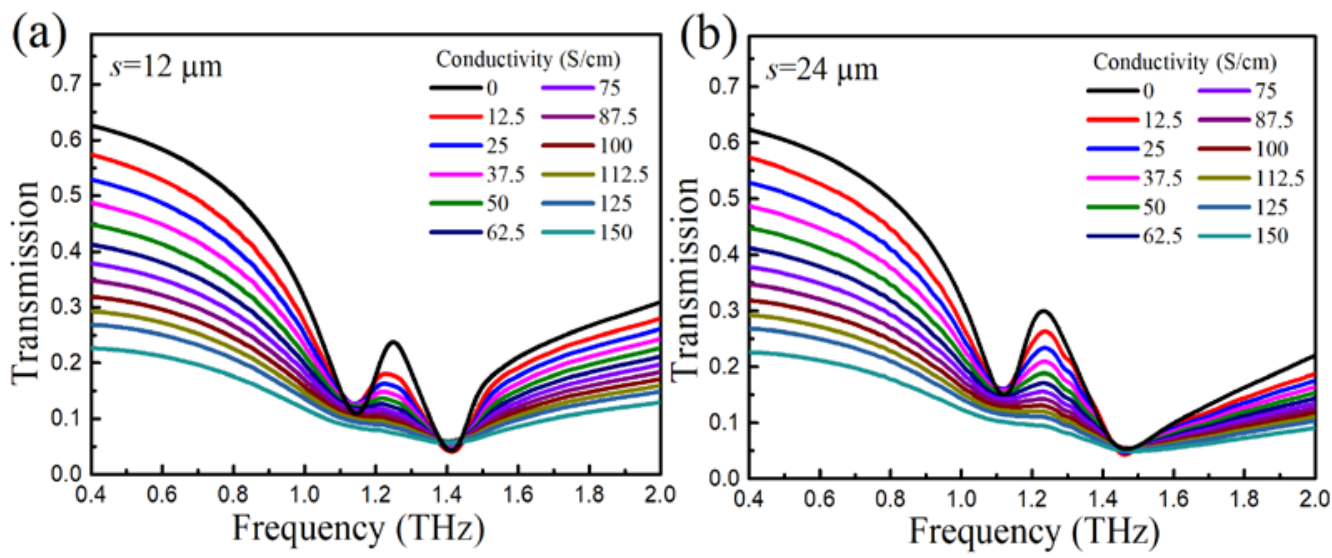

Figure S6. Simulated transmission spectra of two hybrid samples with $s$ of $12 \mu \mathrm{m}$ (a) and 24 $\mu \mathrm{m}$ (b) by changing the photoconductivity of photoexcited graphene/GaAs layer.

\section{Definition of $A_{\text {off }}$ and $A_{\text {on }}$}

Here, we define the peak to dip amplitude transmission modulation depth of MIT as $\left[\left(\mathrm{A}_{\text {off }}-\mathrm{A}_{\mathrm{on}}\right) / \mathrm{A}_{\mathrm{off}}\right] \times 100 \%$, where $A_{\text {off }}=T_{\text {peak }}^{\text {off }}-T_{\text {dip }}^{\text {off }}$ and $A_{\text {on }}=T_{\text {peak }}^{\text {on }}-T_{\text {dip }}^{\text {on }}$ stand for the transmission difference in "off" and "on" states of optical pump, respectively, as presented in Figure S7.

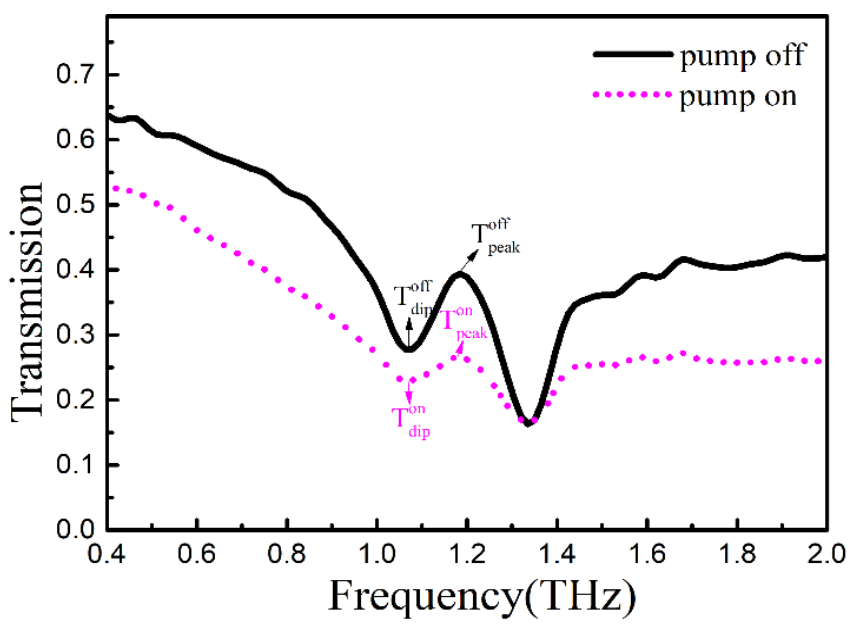

Figure S7. Definition of $T_{\text {peak }}^{\text {off }}$, $T_{\text {dip }}^{\text {off }}, T_{\text {peak }}^{\text {on }}$, and $T_{\text {dip }}^{\text {on }}$. 


\section{Polarization conversion curves}

Such terahertz metamaterial with metallic bar structure has the polarization modulation effect, as given in Figure S8. For the UBLD/GaAs structures without graphene, our simulated results exhibit there is no polarization conversion effect when the metallic bar direction is along the incident $\mathrm{THz}$ polarization ( $x$-direction), i.e., $0^{\circ}$. This implies that the horizontal metal strip array could not change the polarization state of $\mathrm{THz}$ wave, only filter and modulate the $\mathrm{THz}$ wave at specific frequency. If the bar direction is perpendicular to the $\mathrm{THz}$ polarization, i.e., $90^{\circ}$, the transmission spectrum of $x$ component is a straight line without the dipole resonant absorption, showing the value is about $70 \%$ equal to the transmittance of the substrate. The $y$ component transmission is zero without polarization conversion. When the metastructure is tilted by $45^{\circ}$, the maximum cross-polarization conversion efficiency can be achieved and the HFRM conversion reaches about half of the substrate transmittance. After the deposition of graphene overlayer, although the resonance spectrum at $0^{\circ}$ is greatly modulated by the introduction of graphene, there is still no polarization conversion. It is worth noting that the transmission curves of $x$ component without and with graphene have little difference at $90^{\circ}$, indicating the individual graphene layer shows high transmittance at $\mathrm{THz}$ band, which is verified by our experiment. Similarly, the maximum polarization conversion efficiency is approached at $45^{\circ}$ accompanied by the high value at LFRM. Our simulated results exhibit such hybrid structure has great potential for THz polarizer application. 
(a)

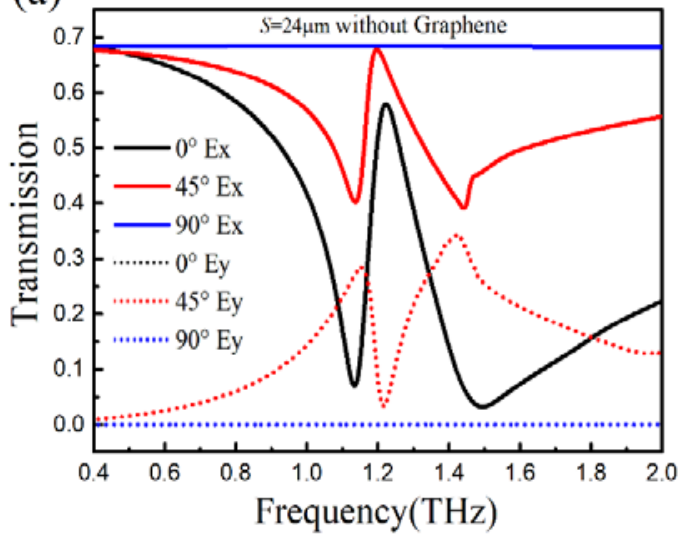

(b)

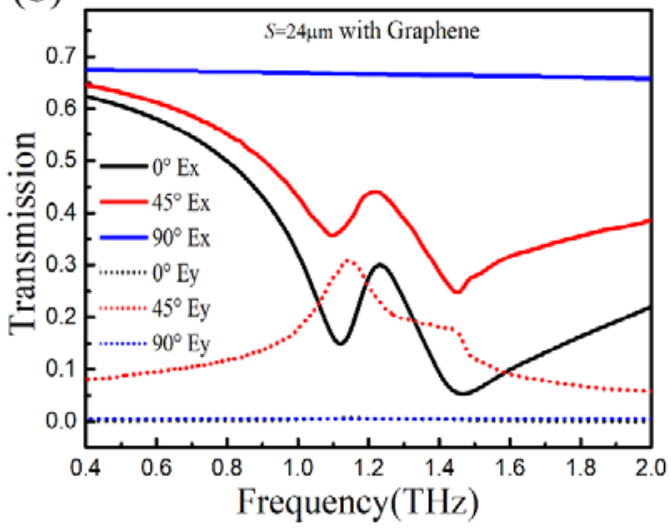

Figure S8. Simulated transmission spectra of $x$-polarized (solid lines) and $y$-polarized (dotted lines) components for $s$ of $24 \mu \mathrm{m}$ without (a) and with (b) graphene overlayer at rotation angle of $0^{\circ}, 45^{\circ}$, and $90^{\circ}$, respectively.

\section{Derivation formulas of complex THz photocoductivity}

Separating the real and imaginary parts of Drude-smith model, we can have the complex conductivity with

$$
\sigma_{1}(\omega)=\frac{n^{*} e^{2} \tau / m}{\left(1+\omega^{2} \tau^{2}\right)}\left[1+\frac{c\left(1-\omega^{2} \tau^{2}\right)}{\left(1+\omega^{2} \tau^{2}\right)}\right]
$$

and

$$
\sigma_{2}(\omega)=\frac{n^{*} e^{2} \tau / m \cdot \omega \tau}{\left(1+\omega^{2} \tau^{2}\right)}\left[1+\frac{2 c}{\left(1+\omega^{2} \tau^{2}\right)}\right]
$$

\section{Description of THz response intensity}

$$
C_{1}=\frac{a_{1}\left(\omega_{2}{ }^{2}-\omega^{2}+i \gamma_{2} \omega\right)-a_{2} v_{12}}{\left(\omega_{1}^{2}-\omega^{2}+i \gamma_{1} \omega\right)\left(\omega_{2}{ }^{2}-\omega^{2}+i \gamma_{2} \omega\right)-v_{12}{ }^{2}}
$$

We can derive equation (3) by substituting $x_{1}=C_{1} e^{i \omega t}$ and $x_{2}=C_{2} e^{i \omega t}$ into the coupled Lorentz oscillator model in the text. $\left|C_{1}\right|$ represents the THz response intensity of ULDB metamaterials. 


\section{References}

(1) Shi, S. F.; Tang, T. T.; Zeng, B.; Ju, L.; Zhou, Q.; Zettl, A. Controlling Graphene Ultrafast Hot Carrier Response from Metal-like to Semiconductor-like by Electrostatic Gating. Nano Lett. 2014, 14, 1578-1582.

(2) Frenzel, A. J.; Lui, C.H.; Shin, Y.C.; Kong, J.; Gedik, N. Semiconducting-to-metallic Photoconductivity Crossover and Temperature-dependent Drude Weight in Graphene. Phys. Rev. Lett. 2014, 113, 056602.

(3) Jensen, S.A.; Mics, Z.; Ivanov, I.; Varol, H. S.; Turchinovich, D.; Bonn, M. Competing Ultrafast Energy Relaxation Pathways in Photoexcited Graphene. Nano Lett. 2014, 14, 5839-5845.

(4) Hafez, H. A.; Lévesque, P. L.; Al-Naib, I.; Dignam, M. M.; Chai, X.; Choubak, S. Desjardins, P.; Martel, R.; Ozaki, T. Intense Terahertz Field Effects on Photoexcited Carrier Dynamics in Gated Graphene. Appl. Phys. Lett. 2014, 107, 251903.

(5) Docherty, C. J.; Lin, C.; Joyce, H. J.; Nicholas, R. J.; Herz, L. M.; Li, L.; Johnston, M. B. Extreme Sensitivity of Graphene Photoconductivity to Environmental Gases. Nat. Commun. 2012, 3, 1228. 